\title{
Phylogenetic Analysis of Mycoplasmas Based on Hsp70 Sequences: Cloning of the dnaK (hsp70) Gene Region of Mycoplasma capricolum
}

\author{
MIZIED FALAH AND RADHEY S. GUPTA* \\ Department of Biochemistry, McMaster University, Hamilton, Ontario L8N 3Z5, Canada
}

\begin{abstract}
A 5.0-kb region containing the $h s p 70$ (dnaK) gene was cloned from Mycoplasma capricolum and sequenced. In addition to the $h s p 70$ gene, this sequence region also contained the complete sequences for the $\operatorname{grp} E$ and $\operatorname{orf} A$ genes and partial sequences for the $c l p B$ and $d n a J$ genes. The order of the above gene sequences in the cloned fragment was found to be clpB-orfA-grpE-hsp70-dnaJ, which is similar to the order seen in various other gram-positive groups of bacteria. The Hsp70 homologs from two mycoplasma species, Mycoplasma capricolum and Mycoplasma genitalium, contain a number of sequence signatures, including the absence of a large insert in the N-terminal quadrant, that are characteristics of the homologs from gram-positive bacteria and archaebacteria. A detailed phylogenetic analysis based on $\mathrm{Hsp70}$ sequences was also performed. In neighbor-joining and parsimony trees based on Hsp70 sequences, both mycoplasma species branched with the low-G+C-content gram-positive group of bacteria (e.g., Lactobacillus and Erysipelothrix species) in $87 \%$ and $96 \%$ of the bootstrap replicates, respectively, indicating their close evolutionary relationship to this group. The phylogenetic trees based on Hsp70 sequences show a polyphyletic branching of archaebacteria with the gram-positive species, which is statistically strongly favored.
\end{abstract}

Mycoplasma species are the smallest free-living microorganisms known. They lack a typical eubacterial cell wall, periplasmic space, and an outer membrane $(20,26,27)$. Their genomes are far smaller than the typical genomes of prokaryotes (they are one-sixth to one-third the size of the Escherichia coli genome) and approach the theoretical size limit for genomes of free-living organisms $(11,20,27)$. Mycoplasma species are distinct because of their genome size and their strict requirement for exogenous sterol $(20,26)$. The peculiar characteristics of mycoplasma species have aroused considerable interest concerning the evolution of these organisms. Phylogenetic studies based on 16S rRNA sequence data have indicated that the mycoplasmas branch with the low-G + C-content subgroup of gram-positive eubacteria, namely, the Lactobacillus group (20, $30,32)$. Due to the limited genome size, many gene functions are absent from mycoplasmas; however, heat shock treatment of Mycoplasma capricolum cells has been reported to cause increased synthesis of seven distinct protein bands, including a $70-\mathrm{kDa}$ protein which reacts with antibodies to the $E$. coli DnaK protein (5). In our recent work we have been utilizing sequence data for the Hsp 70 family of proteins to examine the evolutionary relationships among different groups of species $(9,14-16)$. Because of their large size and high degree of sequence conservation (the most conserved proteins found in any species) the Hsp 70 proteins are well suited for examining deep phylogenetic relationships. Phylogenetic analyses based on Hsp 70 sequences and several other protein sequences have revealed a number of important differences from rRNA-based phylogenies $(1,2,4,12,14,15,29)$. In particular, a close evolutionary relationship between archaebacteria and the gram-positive group of bacteria has been suggested by these studies $(12,15)$. In view of these results and the limited genome size of mycoplasmas, we cloned the $h s p 70$ gene from $M$. capricolum to confirm the evolutionary relationship of this organism with other species. In this paper we present the

* Corresponding author. Phone: (905) 525-9140, ext. 22639. Fax: (905) 522-9033. results of cloning and sequencing studies of $h s p 70$ and a number of adjoining genes from $M$. capricolum. Recently, the complete genomic sequence of another mycoplasma species, $M y$ coplasma genitalium, was reported (11). The evolutionary relationship of mycoplasmas to other prokaryotic species was examined in this study.

\section{MATERIALS AND METHODS}

PCR. Genomic DNA from $M$. capricolum was kindly provided by P. M. Gillevet, George Mason University, Fairfax, Va. Degenerate oligonucleotide primers with opposite orientations for two conserved regions of Hsp70 sequences were synthesized at the Central Facility of the Institute of Molecular Biology, McMaster University, Hamilton, Canada. A forward primer (5'-CARGCNAC NAARGAYGCNGG-3') and a reverse primer (5'-GCNACNGCYTCRTCNG GRTT-3') (where $\mathrm{N}=\mathrm{A}, \mathrm{C}, \mathrm{G}$, or $\mathrm{T} ; \mathrm{Y}=\mathrm{C}$ or $\mathrm{T}$; and $\mathrm{R}=\mathrm{A}$ or $\mathrm{G}$ ) were made for sequence regions QATKDAG and NPDEAVA, respectively (13). PCR amplification in which $M$. capricolum genomic DNA and the primers described above were used was carried out as described previously (13). After 30 cycles, an expected $0.65-\mathrm{kb}$ fragment which showed specific amplification was cloned and sequenced to ascertain its Hsp70-related nature and uniqueness. The cloned PCR fragment was used as a probe in Southern blots and colony-screening studies. To isolate a genomic clone for Hsp70, the genomic DNA was digested with a number of restriction enzymes and resolved on a $1 \%$ agarose gel. The region which hybridized to the PCR probe in the Southern blots was excised, and a library made from this DNA was screened with the ${ }^{32} \mathrm{P}$-labelled cloned $M$. capricolum PCR probe. The DNA was completely sequenced after nested sets of deletions were made with a T7 sequencing kit (Pharmacia).

Phylogenetic analyses. The GenBank accession numbers of various Hsp70 homologs have been published previously (15). A multiple alignment of the Hsp70 family of sequences has been described previously $(9,13,16)$, and the sequences for mycoplasma $\mathrm{Hsp} 70$ s were added to this alignment. A phylogenetic analysis was carried out by using the sequence region corresponding to the region between amino acid 6 (beginning with IIGID) and amino acid 512 (ending with KNKAE) in the $M$. capricolum sequence, which could be aligned with minimum ambiguity in all Hsp70 homologs $(9,14-16)$. Phylogenetic analyses of sequence data were performed by using the programs BOOT, NEIGHBOUR, PROTPARS, PROTDIST, and CONSENSE from the PHYLIP version 3.5 program package $(10,28)$.

\section{RESULTS}

Cloning of the $h s p 70$ gene. The $h s p 70$ gene region from $M$. capricolum was cloned by using a PCR-based approach that has been successfully employed in our laboratory for cloning hsp70/dnaK from a variety of prokaryotic and eukaryotic spe- 


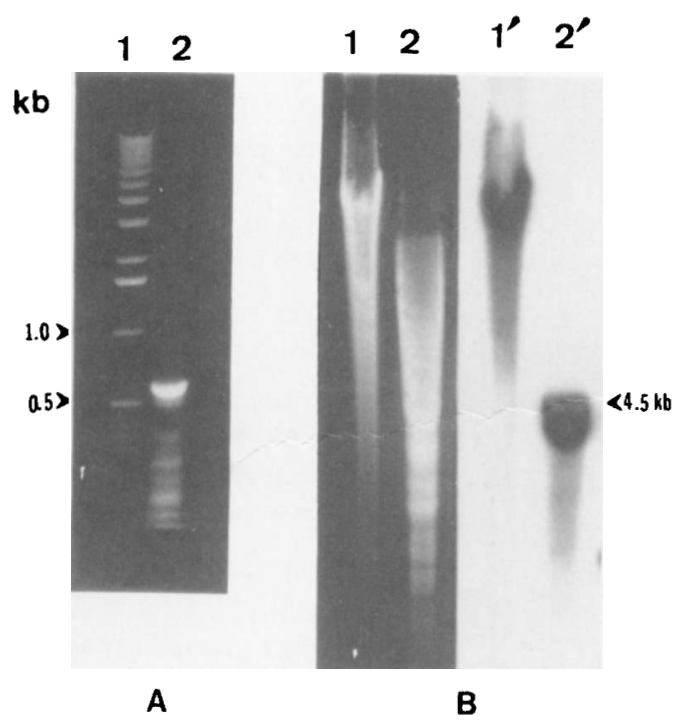

FIG. 1. Cloning of the $M$. capricolum hsp70 gene. (A) Agarose gel electrophoresis of the PCR product of $M$. capricolum genomic DNA when degenerate oligonucleotide primers for conserved regions of $h s p 70$ genes were used. Lane 1 , molecular size markers: lane 2, amplified 0.65 -kb PCR fragment used as a probe in panel B. (B) Southern blot analyses of genomic DNA. Lanes 1 and 2, DNA digested with Bam $\mathrm{HI}$ and $E_{c o} \mathrm{RI}$, respectively, and stained with ethidium bromide; lanes $1^{\prime}$ and $2^{\prime}$, results of hybridization of lanes 1 and 2 , respectively, with the PCR probe.

cies $(9,13-16)$. PCR amplification with primers for two highly conserved regions of $\mathrm{Hsp} 70$ led to the isolation of a $0.65-\mathrm{kb}$ fragment (Fig. 1A), whose sequence was unique but showed a high degree of similarity to the Hsp70 family of proteins (data not shown). To isolate a genomic clone for Hsp70, M. capricolum genomic DNA was digested with BamHI and EcoRI endonucleases and resolved on an agarose gel (Fig. 1B). Southern blot analyses in which the cloned PCR probe was used revealed specific hybridization to a $4.5-\mathrm{kb}$ fragment for the EcoRI-digested DNA (Fig. 1B, lane $2^{\prime}$ ). To clone this fragment, the DNA region between 4 and $5 \mathrm{~kb}$ from EcoRI-digested DNA was subcloned in plasmid pGEM-7z(+). After transformation, the resultant library was screened by using the cloned PCR probe. The positive clones contained an approximately $4.5-\mathrm{kb}$ insert which hybridized to the PCR probe. Sequencing of the insert revealed that some sequence from the $3^{\prime}$ end of the $h s p 70$ gene (200 bp) was missing. To clone the missing region, genomic DNA was digested with DraI and hybridized to a probe from the $3^{\prime}$ end of the original clone (probe P2) (Fig. 2). A 1.8-kb fragment which hybridized to this probe was cloned, and it contained the $3^{\prime}$ end of the $h s p 70$ gene. The 4.5- and 1.8-kb fragments were completely sequenced. Translation of the $5.1-\mathrm{kb}$ region that was spanned by these two fragments revealed that in addition to the $h s p 70$ gene sequence, this region also contained either complete or partial sequences for the following genes: $\operatorname{clp} B, \operatorname{orf} A, \operatorname{grp} E$, and $d n a J$. The nucleotide sequence and the open reading frames corresponding to the above proteins that are present in this fragment are shown in Fig. 3. This sequence has been deposited in GenBank database under accession number U51235. A partial sequence of some of these genes from $M$. capricolum was also reported recently by Bork et al. (3) as part of its genomic sequence.

The $\mathrm{G}+\mathrm{C}$ content of the $M$. capricolum DNA sequence region was found to be $25.8 \mathrm{~mol} \%$, which is consistent with the previously published value (26). The codon usage in the coding region revealed that there was a strong preference for $A$ or $T$ at the third position. Another novel feature of the codon usage is that the codon UGA, which normally codes for a stop codon, was found to code for tryptophan. This unusual use of UGA, as well as a number of other codons (e.g., CGG is an unassigned codon instead of coding for arginine), in $M$. capricolum is well known from earlier studies $(23,25)$ and appears to have resulted from A/T-induced selection pressure.

Sequence alignment and phylogenetic analyses. By the time that we completed the cloning and sequencing of the $h s p 70$ gene region from $M$. capricolum, the sequence of another mycoplasma, $M$. genitalium, became available as a result of cloning of the entire genome of this species (11). $M$. genitalium is a member of the Mycoplasma pneumoniae group and is thought to have the smallest genome $(580 \mathrm{~kb})$ among eubacterial species $(11,20)$. Therefore, both mycoplasma Hsp70 sequences were utilized in further analyses. A multiple alignment of the
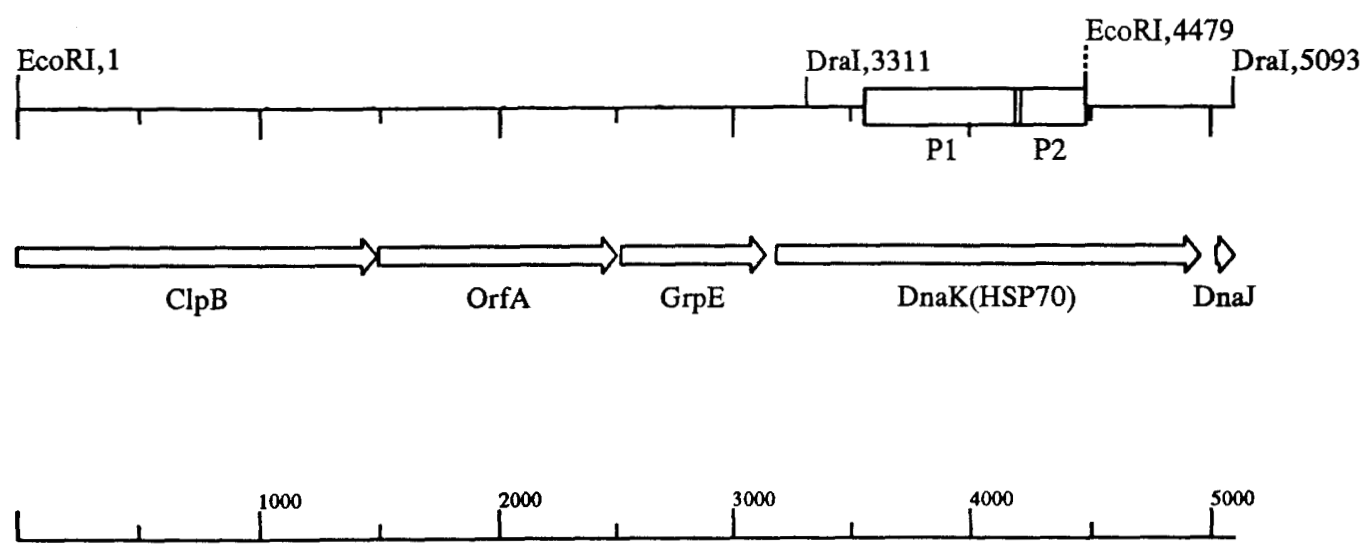

\section{(5098 bp)}

FIG. 2. Gene arrangement and restriction map of the cloned region. The positions of probes $\mathrm{P} 1$ and $\mathrm{P} 2$, which were used to isolate the EcoRI-EcoRI (4.5-kb) and DraI-DraI $(1.8-\mathrm{kb})$ fragments, respectively, are shown. The arrows indicate the open reading frames corresponding to the $c l p B$, orf $A$, grpE, $h s p 70 / d n a K$, and $d n a J$ proteins. 
Clp B

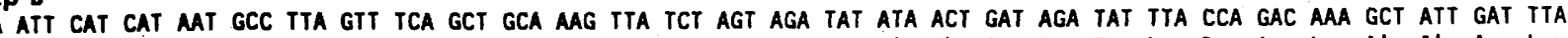
Ile his his Asn ala leu Val Ser ala Ala lys Leu Ser Ser arg tyr Ile Thr Asp Arg tyr Leu Pro Asp Lys Ala Ile Asp Leu

90 GTT GAT GAA GCT TGT GCT TCT ATT AAA ACA GAA TTA GCA AGT ATT CCA ATt GAA CTA GAT CAA GTA AAT AGA AAA GTA ATG CAA TTA GAA Val Asp Glu Ala Cys Ala Ser Ile Lys Thr glu leu Ala Ser Ile Pro ile glu Leu Asp gln Val Asn Arg Lys Val Met Gln Leu glu

180 ATT GAA ACT TCA GCT TTA GAA AAA GAA AAA GAT GAT AAA TCT AAA GAA AGA TGA CAA GAA GCT AAA AAA GAA TIA GAT AGT TTA AAA ATT Ile glu Thr Ser Ala Leu Glu Lys Glu Lys Asp Asp Lys Ser Lys Glu Arg Trp Gln Glu Ala Lys Lys Glu Leu Asp Ser Leu Lys Ile

270 GM CM GCT ACT TTA MT MM MM TGA GAA MAA GAA AAA GAA GAA TIA AGT AAA ATT AAT TTG GTA AAA TCA AGC ATT GAA AAT TTA AAA glu Gln Ala Thr Leu Asn lys lys trp glu Lys Glu Lys Glu Glu Leu Ser Lys Ile Asn Leu Val Lys Ser Ser Ile Glu Asn Leu Lys.

360 CM GAA TTA GAA ACA GCT CA AAC GAT GGA AAT TAT AAA AGA GCT GGA GAA ATT AAA TAC TCA TTA TTA CCA TCA CTT GAA AAA AGC TTA Gln Glu Leu Glu Thr Ala Gln Asn Asp Gly Asn Tyr Lys Arg Ala Gly Glu Ile Lys Tyr Ser Leu Leu Pro Ser Leu Glu Lys Ser Leu

450 GCT TTA ITT GAA ACA CAA ACT GGA GCA AAA ATG ATT TCA GAA GAA GTA ACT GAA CAA GAA ATT GCA AAA GTT GTT TCT AAA TCA ACA GGA Ala leu Phe Glu Thr Gln thr Gly Ala Lys Met Ile Ser Glu Glu Val Thr Glu Gln Glu ile Ala Lys Val Val Ser Lys Ser Thr Gly

540 ATT TTA GTT GAT MA TTA ATT TCT TCA GAA AAA GAA AGA CTT ITA AAT CTT GAA GAT CTA TTA AAA AAA TAT GTT AAA GGT CAA GAT CAA Ile Leu Val Asp Lys Leu Ile Ser Ser Glu Lys Glu Arg Leu Leu Asn Leu Glu Asp Leu Leu Lys Lys Tyr Val Lys Gly Gln Asp Gln

630 GCT ATT AAA GCA GTA ACT TCA GCA ATT ATG AGA AGT AGA AGT GGA ATT AAA AAT CCA GAT AAA CCA ATT GGT AGT TTT CTA TTT TTC GGA Ala Ile Lys Ala Val Thr Ser Ala Ile Met Arg Ser Arg Ser Gly Ile Lys Asn Pro Asp Lys Pro Ile Gly Ser Phe Leu Phe Phe Gly

720 CCA ACT GGA GTT GGA AAA ACT GAA GTT GCA AGA AGT TTA GCA GAT ATT TTA TIT AAT TCA CCG AAA AAA ATG ATC AGA CTT GAT ATG AGT Pro Thr Gly Val Gly Lys Thr Glu Val Ala Arg Ser Leu Ala Asp Ile Leu Phe Asn Ser Pro Lys Lys Met Ile Arg Leu Asp Met Ser

810 GAA TAT ATG GAA AAG CAT TCT GTT GCT AAA TTG ATT GGT GCT CCT CCT GGA TAT GTT GGG TAT GAA GAA GGA GGA AGA TTA ACT GAA GCT Glu Tyr Met Glu Lys his Ser Val Ala Lys Leu Ile Gly Ala Pro Pro Gly Tyr Val Gly Tyr Glu Glu Gly Gly Arg Leu Thr Glu Ala

900 GTA AGA AGA AAT CCT TAT TCA ATT ATT TTA TTT GAT GAA ATT GAA AAA GCT CAT AGT GAT GTG TTT AAC ATC TTA TTA CAA ATA CTA GAT Val Arg Arg Asn Pro Tyr Ser Ile Ile Leu Phe Asp Glu Ile Glu Lys Ala His Ser Asp Val Phe Asn Ile Leu Leu Gln Ile Leu Asp

990 GAT GGA AGA TTA ACA GAT TCA TTA GGA AAA ACT ATT GAT TTT AAA AAT ACA ATT ATT GTT ATG ACT TCA AAT ATA GCT AGT CAA TAT TTA Asp Gly Arg Leu Thr Asp Ser Leu Gly Lys Thr Ile Asp Phe Lys Asn Thr Ile Ile Val Met Thr Ser Asn Ile Ala Ser Gln Tyr Leu

1080 TTA ACT TCA GAT GAT TTT GTA CAA ATT GAT GAT CAA AAG ATT CAA GCA GAA TTA AAT CAA ACT TTT AGG CCT GAA TTT TTA AAT AGA ATT Leu Thr Ser Asp Asp Phe Val Gln Ile Asp Asp Gln Lys Ile Gin Ala Glu Leu Asn Gln Thr Phe Arg Pro Glu Phe Leu Asn Arg Ile

1170 GAT AAT ATT GTT TAT TTT AAT GCT TTA TCA GTA CAA ACA ATT GGC GAA ATT GTA GAT AAA CTT TTA GAT GAA TTA ATT ACA AGA TTA CAA Asp Asn Ile Val Tyr Phe Asn Ala Leu Ser Val Gln Thr Ile Gly Glu Ile Val Asp Lys Leu Leu Asp Glu Leu Ile Thr Arg Leu Gln

1260 GAT GAA CAA AAC TAT TTT ATT AAT TTT TCA GAA GAA GCT AGA AAT AAA ATT ATT AAT GAA GGT TAT GAT AGG TTA TTT GGA GCA AGA CTA Asp Glu Gln Asn Tyr Phe Ile Asn Phe Ser Glu Glu Ala Arg Asn Lys Ile Ile Asn Glu Gly Tyr Asp Arg Leu Phe Gly Ala Arg Leu

1350 TTA AAA GAT ATA TTG AAA AAA ATA ATT GAA ACT TTA ATA GCG CAT TAT ATT ATT AGT GGT TTG ATA AGT GAA AGT ACT AGA TAT CTA ATT Leu Lys Asp Ile Leu Lys Lys Ile Ile Glu Thr Leu Ile Ala His Tyr Ile Ile Ser Gly Leu Ile Ser Glu Ser Thr Arg Tyr Leu Ile Stop Orfa

1440 GAT GTT AAA AAT AAC CAA TTT ATT ITA GAA GAA TTT AAA CAA TTT AAT TAA TIT T ATG TTA ACT AAA AGA CAA GTT AAA ATT TTA CAA Asp Val Lys Asn Asn Gln Phe Ile Leu Glu Glu Phe Lys Gin Phe Asn ...

1528 ACT ATT GTT GAA GAA TTT ATT AAA ACT AAT CAA CCA GTT GGA TCG AAA AGA ATT TTA GAA CTA TTA GAT ATT AAA ATA TCT TCA GCA ACA Thr Ile Val Glu Glu Phe Ile Lys Thr Asn Gin Pro Val Gly Ser Lys arg lle Leu Glu Leu Leu Asp Ile Lys Ile Ser Ser Ala Thr

1618 ATT AGA MAT GAA TCA GCT ATT TTA GAA CAT GAA GGT TAT TTA GAA AAA CAA CAC ACT TCA AGT GGA AGA ACA CCT TCA ACT AAA GGC TAT Ile Arg Asn Glu Ser Ala lle Leu Glu His Glu Gly Tyr Leu Glu Lys Gln His Thr Ser Ser Gly Arg Thr Pro Ser Thr Lys Gly Tyr

1708 AGA TAT TAT GTT GAT AAT ATT ATG AAA CTA GAT TCA GCT GAT TAT ACT AGA TTA AAA ATT TAT TTA AAC CAG ITA TTA GAT TTA AGA AAA Arg Tyr Tyr Val Asp Asn Ile Met Lys Leu Asp Ser Ala Asp Tyr Thr Arg Leu Lys Ile Tyr Leu Asn Gln Leu Leu Asp Leu Arg Lys

1798 TAT GAT ATT GAT AMA ACG ATT AAT TAT GCT AGT GAA ATT ATT AGT GAA TTA ACT AAA ATG ACA GCA GTT GTA ATT AAA AGC AAA AAT ATA Tyr Asp Ile Asp Lys Thr Ile Asn Tyr Ala Ser Glu Ile Ile Ser Glu Leu Thr Lys Met Thr Ala Val Val Ile Lys Ser Lys Asn Ile

1888 AAA AAT ATA AAG TTA AAA AAA ATT GAA CTA ATA TTA TTA TCA GAA TTT TTA GCA AGT GTA TTA TTT ATT TTT TCT GAT GGT GAT GTG CAA Lys Asn Ile Lys Leu Lys Lys Ile Glu Leu Ile Leu Leu Ser Glu Phe Leu Ala Ser Val Leu Phe Ile Phe Ser Asp Gly Asp Val Gln

1978 AAT AAA ATG TTT AAT TTA AAA GAT ATT TCT TTA TCT GAT TTA AAA ATT GCT ATT AAA TTA TTT TCA GAT TTT TTA GTT GAT GTT AAA TTA Asn Lys Met Phe Asn Leu Lys Asp Ile Ser Leu Ser Asp Leu Lys Ile Ala Ile Lys Leu Phe Ser Asp Phe Leu Val Asp Val Lys Leu

2068 GAT GAA ATA GAT CAA TAT TTA AAT GAT TTA AAG CAT CAA TTA TCT ITA AGT ATT AAA CAA TAT GAC TAT GTT TTA AAC ACA TTT ATA AAT Asp Glu Ile Asp Gln Tyr Leu Asn Asp Leu Lys His Gln Leu Ser Leu Ser Ile Lys Gln Tyr Asp Tyr Val Leu Asn Thr Phe Ile Asn

FIG. 3. Nucleotide sequence of the cloned region and deduced amino acid sequences of the various encoded proteins. The start points and the ends of different genes are indicated. 
2158 ACT ATT TTA GAA TCA AAA AAT GAA CAA AAA GAA ACT CAT GGA ATG AGA TAT ATG TTA GAA AAT CCT GAG TTT AAT GAT ACT AAT AAA ITA Thr Ile Leu Glu Ser Lys Asn Glu Gln Lys Glu Thr His Gly Met Arg Tyr Met Leu Glu Asn Pro. Glu Phe Asn Asp Thr Asn Lys Leu

2248 MA AAT GCA GTT AAA TTA GTT GAA CAA TTA TCT CCT TTT GAT TGA TTT AAT ATT GCT TAT GAA TCT AAT AAA AAT ATG AAT AAA ATA GCA Lys Asn Ala Val Lys Leu Val Glu Gln Leu Ser Pro Phe Asp Trp Phe Asn Ile Ala Tyr Glu Ser Asn Lys Asn Met Asn Lys Ile Ala

2338 ATt MM ATT GGT AAT GA ATC GAC CAA ATA AAT GAT GAT ATT TCA ATG ATT GCT ACA GAA TTA AAA ATT GGT AAT TCT TCT ACT GTT TTA Ile Lys Ile Gly Asn Glu Ile Asp Gln lle Asn Asp Asp Ile Ser Met Ile Ala Thr Glu Leu Lys Ile Gly Asn Ser Ser Thr Val Leu

S28 ACT TTA GTA GOT CCA

AGA TTA CAA CCA AGT AAA CCA GTT AAT GAA CTT AAT TAT TGA AAT TAT TAA TGCAAAgGa AATtAAA Thr Leu Val Gly Pro lys Arg Gly Arg Leu Gln Pro Ser Lys Pro Val Asn Glu Leu Asn Tyr Trp Asn Tyr -..Grpe

2520 ATG ACT GAA GAA TTA AAA AAT AAA AAA AAT AAT AAA AAC TAT TAT AGT CAA AAT AAA AAT AAA ACT AAA GCT GAA TTT CAA AAA CCT CAT Met Thr Glu Glu Leu lys Asn Lys Lys Asn Asn Lys Asn Tyr Tyr Ser GIn Asn Lys Asn Lys Thr Lys Ala Glu Phe Gln Lys Pro His

2610 GTT AAA AAA AAT CAA TAT TTA AAG TTA AAA ACA AAA CTT GAT ACT GCT TTA TTA GAA GTT CAA AAT TTA AAA GAT TTA AAT GAA ACT TTA Val Lys Lys Asn Gln Tyr Leu Lys Leu Lys Thr Lys Leu Asp Thr Ala Leu Leu Glu Val Gln Asn Leu Lys Asp Leu Asn Glu Thr Leu

2700 M AM GAT ATT GAA TCT GAA AGA CAA CTT AAT TTA GCT GAA ATT AGT AAT TTA ACT AAA AAG TAT AAT CAA AAA GAA ATA GAA ATT CAA Lys Lys Asp Ile Glu Ser Glu Arg Gln Leu Asn Leu Ala Glu Ile Ser Asn Leu Thr Lys Lys Tyr Asn Gln Lys Glu Ile Glu Ile Gln

2790 M TAT GGC GCT AGT AAA TTA GCA AGA GAC TTA ATT CAA CCT TTA GAA ATT TTA AAA AAA GTT GTT AAT GCT CCT AAT AAT AAT GAA GTA Lys Tyr Gly Ala Ser Lys Leu Ala Arg Asp Leu Ile Gln Pro Leu Glu Ile Leu Lys Lys Val Val Asn Ala Pro Asn Asn Asn Glu Val

2880 GTT CTT CGA AAT GTT AAA GGG TTT GAA ATG ATT GTT AGT CAA ATA AAT AAC GTT TTA GAA TCA CAT CAT ATT AAA GCT ATG AAT GTT AAA Val Leu Arg Asn Val Lys Gly Phe Glu Met Ile Val Ser Gln Ile Asn Asn Val Leu Glu Ser his His Ile Lys Ala Met Asn Val Lys

2970 GTT GGT GAT ATG TTT AAT CCA CAT CTT CAT GAT GCT AAT GAA GCA GTT GAA TCA GAT GAA TAT AAA ACA AAT CAA ATT GTT GGT GTT TTA Val Gly Asp Met Phe Asn Pro His Leu His Asp Ala Asn Glu Ala Val Glu Ser Asp Glu Tyr Lys Thr Asn Gln Ile Val Gly Val Leu Stop

3060 AGT GAT GGG TAT ATG ATC CAT GAT AAA GTA CTA ATT TAT GCA ATA GTC AAA GTT GCC AAA ATA AAT AAA AAC AAT AAT TAA AATAAAAAAA Ser Asp Gly Tyr Met Ile His Asp lys Val Leu Jle Tyr Ala Ile Val Lys Val Ala Lys Ile Asn Lys Asn Asn Asn ...

3151 TAGTAAAAAA AGATACAAGg aAgAaAATAA AACC dnak

ATG GCA AAA GAA AAA ATT ATT GGA ATA GAT TTA GGA ACT ACT AAT TCA GTT GTT TCA GTT MET Ala Lys Glu Lys Ile Ile Gly Ile Asp Leu Gly Thr Thr Asn Ser Val Val Ser Val

3245 ATT GAA GGT GGT CAA CCT ATT ATT TTA GAA AAT CCT GAA GGT CAA AGA ACT ACA CCA AGT GTT GTT GCT TTT AAA AAT TCA GAT ATT ATT Ile Glu Gly Gly Gin Pro Ile Ile Leu Glu Asn Pro Glu Gly Gln Arg Thr Thr Pro Ser Val Val Ala Phe Lys Asn Ser Asp Ile Ile

3335 GTT GGT GGA GCT GCT AAA CGT CAA GCT GTA ACT AAT CCA AAT GTT GTT CAA TCA ATA AAA TCA AAA ATG GGA ACT ACT TCT AAA GTT AAT Val Gly Gly Ala Ala Lys Arg Gln Ala Val Thr Asn Pro Asn Val Val Gln Ser Ile Lys Ser Lys Met Gly Thr Thr Ser Lys Val Asn

3425 TTA GAA GGA AAA GAT TAT AGT CCA GAA CAA ATT TCA GCT GAA ATT TTA AGA TAT ATG AAA AAT TAT GCT GAA GCT AAA TTA GGA CAA AAA Leu Glu Gly Lys Asp Tyr Ser Pro Glu Gln Ile Ser Ala Glu Ile Leu Arg Tyr Met Lys Asn Tyr Ala Glu Ala Lys Leu Gly Gln Lys

3515 GTA ACT AAA GCT GTT ATT ACA GTT CCT GCT TAT TTT AAT GAT GCA CAA AGA AAA GCT ACA AAA GAT GCT GGA ACA ATT GCA GGA TTA CAA Val Thr Lys Ala Val Ile Thr Val Pro Ala Tyr Phe Asn Asp Ala Gln Arg Lys Ala Thr Lys Asp Ala Gly Thr Ile Ala Gly Leu Gln

3605 GTT GAA AGA ATT ATT AAT GAA CCA ACT GCC GCT GCT TTA GCT TAT GGA CTA GAT AAA CAA GAT AAA GAA GAA ACA ATT TTA GTT TAT GAT Val Glu Arg Ile Ile Asn Glu Pro Thr Ala Ala Ala Leu Ala Tyr Gly Leu Asp Lys Gln Asp Lys Glu Glu Thr Ile Leu Val Tyr Asp

3695 TTA GGA GGA GGA ACT TTT GAT GTT TCT ATT CTA GCT ATT GGT GGT GGA AGT TTT GAT GTT ATT GCA ACT AGT GGA AAT AAT AAA TTA GGT Leu Gly Gly Gly Thr Phe Asp Val Ser I le Leu Ala Ile Gly Gly Gly Ser Phe Asp Val Ile Ala Thr Ser Gly Asn Asn Lys Leu Gly

3785 GGA GAT AAT TTT GAT GAA GAA ATT ATC AAA TGA TTA CTA GGT AAA ATT AAA GCT GAA TAC AAT ATT GAT TTA TCT AAA GAA AAA ATG GCT Gly Asp Asn Phe Asp Glu Glu Ile Ile Lys Trp Leu Leu Gly Lys Ile Lys Ala Glu Tyr Asn Ile Asp Leu Ser Lys Glu Lys Met Ala

3875 TTA CAA AGA TTA AAA GAT GAA GCT GAA AAA GCA AAA ATT AAT TTA TCT AGC CAA TTA GAA GTT GAA ATT AAT TTA CCA TTT ATT GCA ATG Leu Gin Arg Leu Lys Asp Glu Ala Glu Lys Ala Lys Ile Asn Leu Ser Ser Gln Leu Glu Val Glu Ile Asn Leu Pro Phe Ile Ala Met

3965 AAT GAA AGT GGA CCA ATT TCT TTT GCA ACT CTA ACA AGA AGT GAA TTT AAC AAA ATT ACA AAA CAT TTA GTT GAC TTG ACT ATT CAA CCA Asn Glu Ser Gly Pro Ile Ser Phe Ala Thr Leu Thr Arg Ser Glu Phe Asn Lys Ile Thr Lys His Leu Val Asp Leu Thr Ile Gln Pro

4055 GTT AAA GAT GCT TTA AGT GCT GCT AAA AAA ACT CCA AGT GAA ATT AAT GAA GTT TTA TTA GTT GGT GGG TCA ACA AGA ATA CCT GCT GTT Val Lys Asp Ala Leu Ser Ala Ala Lys Lys Thr Pro Ser Glu Ile Asn Glu Val Leu Leu Val Gly Gly Ser Thr Arg Ile Pro Ala Val

4145 CAA GAA TTA GTA AAA AGT TTA TTA AAT AAA GAA CCA AAT AGA TCA ATT AAT CCA GAT GAA GTT GTT GCT ATG GGT GCT GCT GTG CAA GGT Gln Glu leu Val Lys Ser Leu Leu Asn Lys Glu Pro Asn Arg Ser Ile Asn Pro Asp Glu Val Val Ala Met Gly Ala Ala Val Gln gly

4235 GGA GTT ITA GCT GGT GAA GTT ACT GAT ATT TTA TTA TTA GAT GTA ACC CCA TTA TCA TTA GGT ATT GAA ACA ATG GGT GGA GTT ATG ACA Gly Val Leu Ala Gly Glu Val Thr Asp Ile Leu Leu Leu Asp Val Thr Pro Leu Ser Leu Gly Ile Glu Thr Met Gly Gly Val Met Thr

$$
\text { FIG. 3-Continued. }
$$

two mycoplasma Hsp70 sequences, various prokaryotic Hsp70 sequences, and a few representative eukaryotic Hsp70 sequences was obtained. This sequence alignment was very similar to that reported previously, with all of the Hsp 70 homologs exhibiting extensive similarity throughout their lengths $(9,16)$. M. capricolum $\mathrm{Hsp} 70$ exhibited $>50 \%$ sequence identity with various prokaryotic homologs, and the minimum level of identity observed for the eukaryotic homologs was in the range of 
4325 MA TTA ATT GAA AGA MT ACT ACA ATT CCA GCA AAA AGA ACT CAA ATT TTT TCA ACT GCA ACA GAT AAT CAA CCA GCT GTT GAT ATT AAT Lys Leu Ile Glu Arg Asn Thr Thr Ile Pro Ala Lys Arg Thr Gln Ile Phe Ser Thr Ala Thr Asp Asn Gln Pro Ala Val Asp Ile Asn

4415 GTT TTA CAA GGT GA AGA GCA ATG GCA GCT GAT AAT AAA TCA TTA GGT CAA TTC CAA CTA ACA GGA ATT CAA CCA GCT CCT AGA GGT ATT Val Leu gln Gly Glu Arg Ala Met Ala Ala Asp Asn Lys Ser Leu Gly Gin Phe Gln Leu Thr Gly Ile Gln Pro Ala Pro Arg Gly Ile

4505 CCA CM ATT GM GTT ACT TTT GAA ATT GAT GCT AAT GGT ATT GTA AGT GTT TCA GCA AAA GAT AAA AAT ACT AAT GAA GAA AAA ACT ATT Pro Gln Ile glu Val Thr Phe Glu Ile Asp Ala Asn Gly Ile Val Ser Val Ser Ala lys Asp Lys Asn Thr Asn Glu Glu Lys Thr Ile

4595 ACt ATt TCA MAT TCA GGA MT TTA AGT GAA GCT GAA GTT GAA AGA ATG ATA AAA GAA GCT CAA GAA AAT GCT GCA AAT GAT GAA GCT AAG Thr Ile Ser Asn Ser Gly Asn Leu Ser Glu Ala Glu Val Glu Arg Met Ile Lys glu Ala Gln Glu Asn Ala Ala Asn Asp Glu Ala Lys

4685 MM MA MAT ATT GAA TTA MM AAT AAA GCT GAA AAC TAT ATT AAC ATT ATT GAA ACT TCA CTA TTA CAA GCT GGT GAT AAA ATT AGT GCT Lys Lys Asn Ile Glu Leu Lys Asn Lys Ala Glu Asn Tyr Ile Asn Ile Ile Glu Thr Ser Leu Leu Gln Ala Gly Asp Lys Ile Ser Ala

4775 GAA CAA AAA GAA CAA TCA CAA AAA ATG ATT GAT GAA ATT AAA GAA CTA GTT AAA AAT GAA AAC TAT GAA GCT TTA GAA CAA AAA ATG GCT Glu Gln Lys Glu Gln Ser Gln Lys Met Ile Asp Glu Ile Lys Glu Leu Val Lys Asn Glu Asn Tyr Glu Ala Leu Glu Gln Lys Met Ala

4865 GAA CTA GAA CAA GCA ATG GCT GCA GCT GCT GAA TTT GCT AAC AAA CAT AAT GAT TCA GAT TCA AAT AAT AAT TCA TCA GAA CAA AAT AAT Glu Leu Glu Gln Ala Met Ala Ala Ala Ala Glu Phe Ala Asn Lys His Asn Asp Ser Asp Ser Asn Asn Asn Ser Ser Glu Gln Asn Asn Stop

4955 TAM TCACACTAAA TATATAAAAA CTAACATTGG TTAGTTTTTG TTTTTATAGA AAAGAAGAGA GAAT ATG AAA AAA AAG GAT TAT TAT GAA GTT TTA GGT MET Lys Lys Lys Asp Tyr Tyr Glu Val Leu Gly

5055 GTA TCA AAA ACT GCT AGT GAA CAA GAA ATC AGA CAA GCT TTA AA 5098 Val Ser Lys Thr Ala Ser Glu Gln Glu Ile Arg Gln Ala Leu

$$
\text { FIG. 3-Continued. }
$$

46 to $47 \%$ over the entire length of the protein. A number of Hsp 70 family protein sequence signatures that are characteristic of the major groups of species have been identified previously (13-16). One prominent sequence signature is a large insert of 23 to 27 amino acids that is present in the $\mathrm{N}$-terminal quadrant of all gram-negative bacteria and eukaryotic Hsp70, but absent in the homologs from archaebacteria and grampositive bacteria. A portion of the sequence alignment that contains this sequence region is shown in Fig. 4. As Fig. 4 shows, as in all of the gram-positive bacteria and archaebacteria, this insert was also absent in the Hsp70 homologs from $M$. capricolum and $M$. genitalium. The lack of this insert in the mycoplasma Hsp70 homologs strongly indicates that there is an evolutionary linkage of these species to the gram-positive eubacteria and archaebacteria. To further verify the evolutionary relationships between mycoplasma and other species, phylogenetic analyses of $\mathrm{Hsp} 70$ sequence data were carried out. In these studies $31 \mathrm{Hsp} 70$ sequences were used, and these sequences included 3 sequences from archaebacteria, $10 \mathrm{se}$ quences from gram-positive eubacteria, 7 sequences from gram-negative eubacteria, 3 sequences of eukaryotic organelle homologs, and 6 sequences of eukaryotic cytosolic and endoplasmic reticulum resident homologs.

A neighbor-joining tree based on these sequences is shown in Fig. 5A. The bootstrap scores for various nodes (based on 100 replicates) are also indicated in Fig. 5A. As in our earlier work $(9,14,15)$, the consensus tree based on Hsp 70 sequences shows a clear distinction between the eukaryotic and prokaryotic species (in $100 \%$ of the bootstrap replicates) and a fairly good distinction (in $87 \%$ of the bootstrap replicates) between the eukaryotes and gram-negative bacteria on one end and the archaebacteria and gram-positive bacteria on the other end (Fig. 5A). The two mycoplasma species grouped together, and they branched with the low-G+C-content gram-positive bacteria in $85 \%$ of the bootstrap replicates.

Parsimony analysis of the sequences described above yielded a single most parsimonious tree requiring a total of 3,965 steps (Fig. 5B). Bootstrap scores for some of the important nodes in this tree are shown in Fig. 5B. As Fig. 5B shows, branching of the archaebacteria with the gram-positive species and branching of the eukaryotic cytosolic homologs with the gram-nega- tive bacteria were strongly supported in $100 \%$ of the bootstrap replicates. The two mycoplasma species again showed a strong affinity for the low-G+C-content gram-positive bacteria, and the bootstrap score of this branching (96 of 100) indicated that this relationship was robust. In the parsimony tree the closest affiliation of the mycoplasma species was with Erysipelothrix rhusiopathiae, which is a human and animal pathogen similar to many mycoplasma species (19). Another difference between the neighbor-joining and parsimony trees is the difference in the branching positions of the eukaryotic cytosolic homologs. The parsimony tree indicates that there is a specific relationship between these homologs and the cyanobacterium-chloroplast group (which has also been observed previously [14, 15], whereas in the neighbor-joining tree a deeper branching of the latter group was observed.

\section{DISCUSSION}

In this paper we describe the cloning and a phylogenetic analysis of an Hsp70/DnaK homolog from $M$. capricolum. The $5.0-\mathrm{kb}$ region surrounding the $h s p 70$ locus that was sequenced in this study has been found to contain either partial or complete sequences of a number of different genes. The genes that are present in the cloned fragment occur in the following order: $\operatorname{clp} B(1.5 \mathrm{~kb}$; partial)-orf $A(1.0 \mathrm{~kb})$-grpE $(0.62 \mathrm{~kb})$ - $h s p 70$ $(1.8 \mathrm{~kb})$-dnaJ $(\approx 0.1 \mathrm{~kb}$; partial). The observed organization of these genes in $M$. capricolum is similar to the organization seen in several gram-positive bacteria (viz., Bacillus subtilis [31], Clostridium acetobutylicum [22], Lactococcus lactis [7], and Staphylococcus aureus [24]) and archaebacteria (viz., Methanosarcina mazei [19);]). In gram-positive bacteria such as $B$. subtilis, the last four genes (i.e., orf $A$-grpE-hsp $70[$ dnaK $]-d n a J)$ form an operon (31), whereas in the gram-negative eubacteria (e.g., E. coli), an orf $A$ gene homolog has not been identified.

The presence of an $h s p 70$ gene in $M$. genitalium and $M$. capricolum, which have minimal genomes (in $M$. genitalium a maximum of 470 protein-encoding sequences are present [11]), is consistent with our understanding that Hsp70 plays an essential role in all cell types $(2,21)$. In addition to Hsp 70 , the other genes that have been identified in the cloned region in $M$. capricolum (viz., dnaJ, grpE, orf $A$, and $c l p B$ ) are also present in 


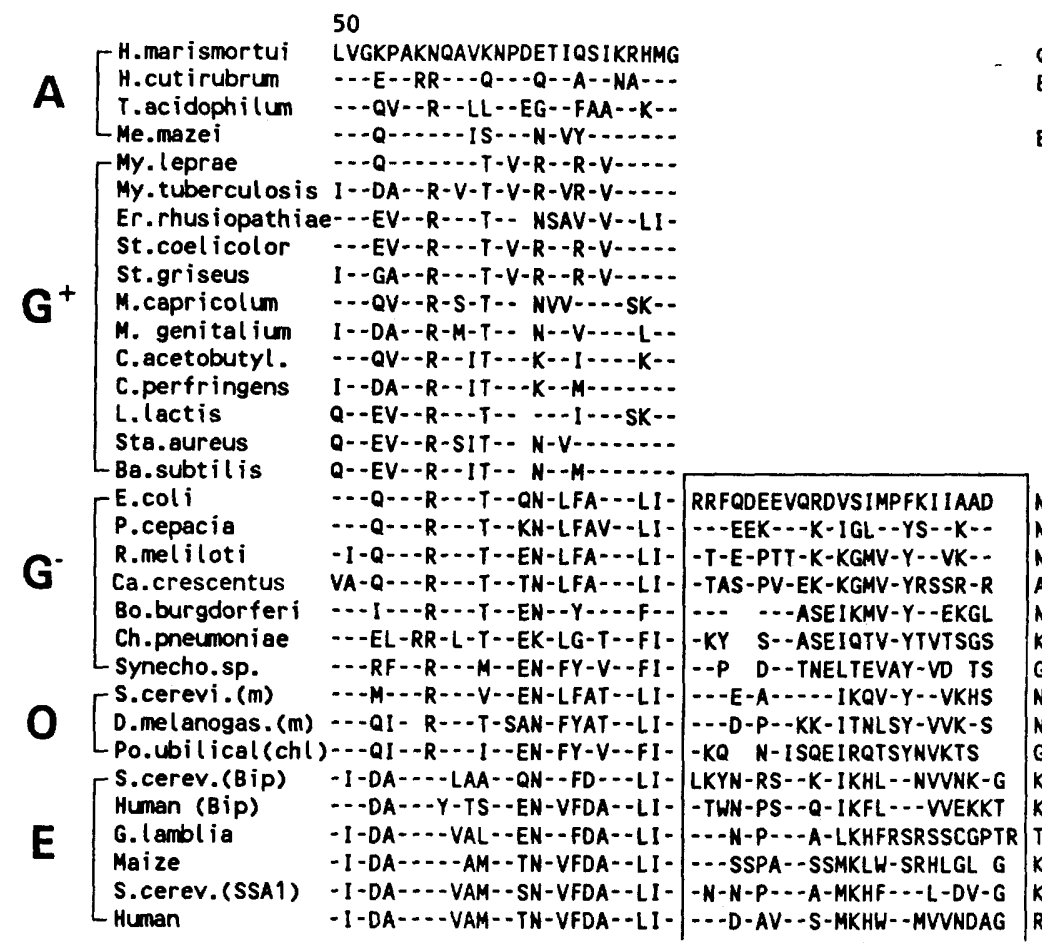

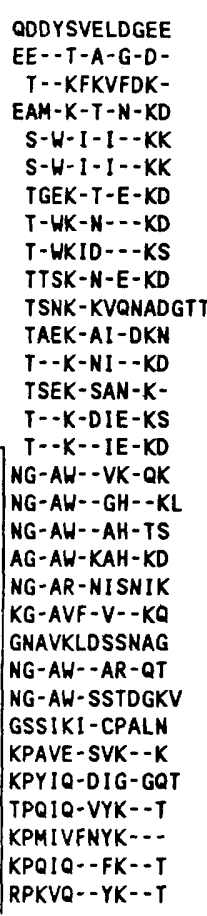

121

YTPEQVSAMILOKIKHDAEEYLGDEIEKAVITV

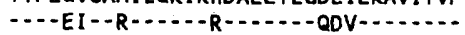
F-OQ-I - -F-.....-K-.-AF--EPVNE-.....

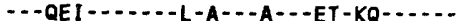

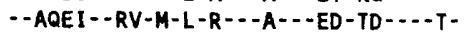
-APEI I-R--M-L-R---A-.-ED-TD-..-T-.- -EI -....-GYM-SY- -D - - EKYT -...... FN-Q-1 -.FV---L-R-.-S-.-EKVTD-...... FN-Q-M--F-..-L-R-R--S-.-EKVTD-..... -S---I--E--RYM-NY--AK--OKVT-....... KELLS-....-Q--SYL-DF--KKI-KK-SR-..... -.-QEI-......-L-A-.-A-.-ETVTQ-..... LS-OEI -......-L-A-.-A-.-EKVTE-..... - - -QEI-....-NL-AT--S-.-EKV-........ -.-OEI--...-NL -NT - -S-.-EKVD-...... ---QE---I-.-HL-SY--S-..-ETVS-...... MA-P-I--EV-L-M-KT--D-.-EPVTE-..... MA-P-..-EAVR-M-KT --D -..EPVTE-..... -S-S-I--..--M-ET--S-.-EKV-....... -S-OE-.-F-..-M-EA--AH--EPVT -....... KOMS-PEI - -AT -T-M-ET--A -.-EKVTE-..... -.-EIG-Q--M-M-ET--A-.-ETVTE-...... KOFA--EI--QV-R-LAE--SK-.-ETVTQ-..... -S-A-IGGFV-N-M-ET--A---KPVKN--V-.-S-S-IG-F--M-M-ET--A--NIPVKN--V-.KDFA--EI--QV-R-LVE--ST---ETVTQ-..... KVF---EI-G---G-M-QI--D---TKVTH--V-.. KTFA--EI---V-T-M-ET--A---KKVTH--V-.. KTF---EI-S-V-T-M-DI-SD--NKVTE-IV-.. KQFAA-EI-S-V-I-M-EI--A--STT-KN--V-.. KNF-.--I-S-V-G-M-ET--S---AKVND--V-.. KSFY--E--S-V-T-M-EI--A-C-KTVTN--V-..

FIG. 4. Part of the Hsp70 sequence alignment showing the relatedness of mycoplasma species to the gram-positive bacteria and archaebacteria. $A, E, G^{-}, G^{+}$, and $\mathrm{O}$ refer to sequences from archaebacteria, sequences from eukaryotes (cytosolic), sequences from gram-negative bacteria, sequences from gram-positive bacteria, and eukaryotic organellar sequences (viz., mitochondrial and chloroplast sequences), respectively. The numbers at the top refer to positions in the Halobacterium marismortui sequence. The region enclosed in a box is the large insert in the N-terminal quadrant present in all Hsp70 homologs from gram-negative bacteria and eukaryotes. Dashes indicate identity with the amino acid in the top line. Abbreviations: Ba., Bacillus; Bo., Borrelia; C., Clostridium; C. acetobutyl., Clostridium acetobutylicum; Ca., Caulobacter; Ch., Chlamydia; D. melanogas., Drosophila melanogaster; E., Escherichia; Er., Erysipelothrix; G., Giardia; H., Halobacterium; L., Lactococcus; M., Mycoplasma; Me., Methanosarcina; My., Mycobacterium; P., Pseudomonas; Po., ubilical, Porphyra umbilicalis; R., Rhizobium; S. cerev., Saccharomyces cerevisiae; St., Streptomyces; Sta., Staphylococcus; Synecho. sp., Synechococcus species; T., Thermoplasma; m, chl, and Bip, mitochondrial, chloroplast, and endoplasmic reticulum resident forms of $\mathrm{Hsp} 70$, respectively.

M. genitalium (11). However, in contrast to the contiguous arrangement of these genes in $M$. capricolum and other bacterial species, these genes have been reported to be widely dispersed in the $M$. genitalium genome, with no two genes exhibiting close proximity (11). The possible significance of this difference in gene organization in $M$. genitalium is not clear at this time.

The sequence features of Hsp70 and the phylogenetic analysis based on the sequence data provide strong evidence that mycoplasma species are evolutionarily closely related to the gram-positive bacteria. Earlier studies on Hsp70 sequences have identified a large insert (between 23 and 27 amino acids long) in the N-terminal quadrant that is present in all of the homologs from gram-negative bacteria and eukaryotic species, but absent in various gram-positive bacteria and archaebacteria (14-16). The sequence alignment of Hsp 70 homologs shows that like the Hsp70 homologs of various other gram-positive bacteria and archaebacteria, the Hsp70 homologs of both mycoplasma species examined also lack this insert. This observation strongly indicates that the mycoplasmas are related to the gram-positive bacterium-archaebacterium group of species.

Detailed phylogenetic analysis based on Hsp70 sequences revealed that the mycoplasma species branch with the low$\mathrm{G}+\mathrm{C}$-content gram-positive bacterial group. The bootstrap scores for this affiliation in the neighbor-joining and parsimony trees indicate that this relationship is reliable. In the parsimony tree, the pathogenic bacterium Enysipelothrix rhusiopathiae was the closest relative of the mycoplasmas. The observed branch- ing of the mycoplasmas with the low-G+C-content gram-positive eubacteria is consistent with the branching observed previously on $16 \mathrm{~S}$ and $5 \mathrm{~S}$ rRNA trees $(6,17,20,30,32)$, the RecA protein sequences $(8,18)$, and a number of other characteristics (20). The fact that different gene and protein sequences, which differ in other important respects $(8,12,5,20,30,32)$, all support a similar evolutionary placement of mycoplasma species strongly indicates that the observed relationship is reliable and that it likely represents the species phylogeny.

One interesting and important difference between the Hsp70 phylogeny and the phylogeny based on 16S rRNA is that the former shows a polyphyletic branching of the archaebacteria with the gram-positive species (Fig. 5). We have previously shown that the observed polyphyletic branching pattern of archaebacteria is statistically strongly supported by results obtained with different phylogenetic methods (14, 15). Furthermore, as discussed in earlier work (12-14), the possibilities that the observed pattern could be due to the use of paralogous sequences, horizontal gene transfer, or tree reconstruction artifacts are considered highly unlikely. Since a similar branching of archaebacteria with gram-positive species is obtained with a number of other conserved proteins, including glutamate dehydrogenase (1) and glutamine synthetase I $(4,29)$, we think that these results may not be unusual but may point to a specific relationship between these two groups. Future studies with additional gene sequences should be helpful in clarifying this relationship. 

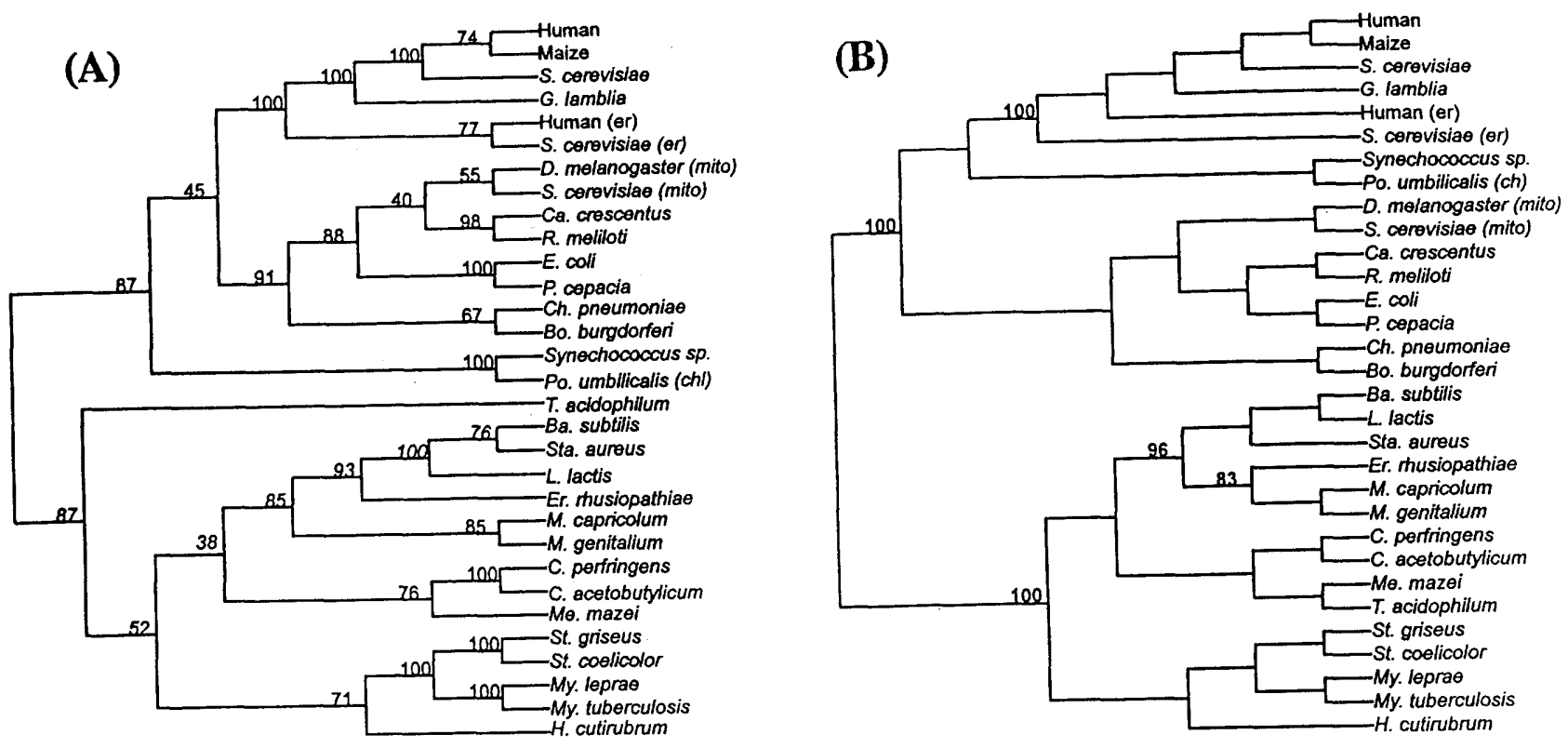

FIG 5. Phylogenetic analysis based on Hsp70 protein sequences. (A) Neighbor-joining distance tree based on the sequence data. The distances were determined by using the PAM 250 matrix. The numbers at the forks indicate the percentages of times that the species to the right grouped together in 100 bootstrap replicates. For abbreviations see the legend to Fig. 4. (B) Maximum-parsimony tree based on the sequences. The bootstrap scores (out of 100) for some of the important nodes are shown. mito, chl, and er refer to the mitochondrial, chloroplast, and endoplasmic reticulum resident forms of Hsp 70, respectively.

\section{REFERENCES}

1. Benachenhou-Lahfa, N., P. Forterre, and B. Labedan. 1993. Evolution of glutamate dehydrogenase genes: evidence for two paralogous protein families and unusual branching pattern of the archaebacteria in the universal tree of life. J. Mol. Evol. 36:335-346.

2. Boorstein, W. R., T. Zeigelhoffer, and E. A. Craig. 1994. Molecular evolution of the HSP70 multigene family. J. Mol. Evol. 38:1-17.

3. Bork, P., C. Ouzounis, G. Cesari, R. Schneider, C. Sander, M. Dolan, W. Gilbert, and P. M. Gilbert. 1995. Exploring the Mycoplasma capricolum genome: a minimal cell reveals its physiology. Mol. Microbiol. 16:955-967.

4. Brown, J. R., Y. Masuchi, F. T. Robb, and W. F. Doolittle. 1994. Evolutionary relationships of bacterial and archael glutamine synthetase genes. J. Mol. Evol. 38:566-576.

5. Dascher, C. C., S. K. Poddar, and J. Maniloff. 1990. Heat shock response in mycoplasma, genome-limited organisms. J. Bacteriol. 172:1823-1827.

6. DeWachter, R., E. Huysmans, and A. Vandenberge. 1985. 5S ribosomal RNA as a tool for studying evolution, p. 115-141. In K. H. Schleifer and E. Stackebrandt (ed.), Evolution of prokaryotes. Academic Press, Inc., New York, N.Y.

7. Eaton, T., C. Shearman, and M. Gasson. 1993. Cloning and sequence analysis of the dnaK gene region of Lactococcus lactis subsp. lactis. J. Gen. Microbiol. 739:3253-3264.

8. Eisen, J. A. 1995. The RecA protein as a model molecule for molecular systematics studies of bacteria: comparison of trees of RecAs and 16S rRNAs from the same species. J. Mol. Evol. 41:1105-1123.

9. Falah, M., and R. S. Gupta. 1994. Cloning of the hsp70/dnaK gene from Rhizobium meliloti and Pseudomonas cepacia: phylogenetic analyses of mitochondrial origin based on a highly conserved protein sequence. J. Bacteriol. 176:7748-7753.

10. Felsenstein, J. 1993. PHYLIP manual, version 3.5. University of Washington, Seattle.

11. Fraser, C. M., J. D. Gocayne, O. White, M. D. Adams, R. A. Clayton, R. D. Fleischmann, J. C. Bult, A. R. Kerlavoge, G. Sutton, J. M. Kelly, J. L. Fritchman, J. F. Weidman, K. V. Small, M. Sandusky, J. Fuhrmann, D. Nguyen, T. R. Utterback, D. M. Saudek, C. A. Phillips, J. M. Merrick, J. F. Tomb, B. A. Dougherty, K. F. Bottom, P. C. Hu, T. S. Lucier, S. N. Peterson, H. O. Smith, C. A. Hutchison III, and J. C. Venter. 1995. The minimal gene complement of Mycoplasma genitalium. Science 270:397-403.

12. Golding, G. B., and R. S. Gupta. 1995. Protein based phylogenies support a chimeric origin for the eukaryotic genome. Mol. Biol. Evol. 12:1-6.

13. Gupta, R. S., and B. Singh. 1992. Cloning of the HSP70 gene from Halobacterium marismortui: relatedness of archaebacterial HSP70 to its eubacterial homologs and a model for the evolution of HSP70 gene. J. Bacteriol. 174: 4594-4605.
14. Gupta, R. S., and G. B. Golding. 1993. Evolution of HSP70 gene and its implications regarding relationships between archaebacteria, eubacteria and eukaryotes. J. Mol. Biol. 37:573-582.

15. Gupta, R. S., and B. Singh. 1994. Phylogenetic analysis of $70 \mathrm{kD}$ heat shock protein sequences suggests a chimeric origin for the eukaryotic cell nucleus. Curr. Biol. 4:1104-1114.

16. Gupta, R. S., K. Aitken, M. Falah, and B. Singh. 1994. Cloning of Giardia lamblia HSP70 homologs: phylogenies based on HSP70 and HSP90 sequences point to gene duplication events accompanying the origin of eukaryotic cells. Proc. Natl. Acad. Sci. USA 91:2895-2899.

17. Hori, H., M. Sawada, S. Osawa, K. Murao, and H. Ishikura. 1981. The nucleotide sequence of 5S rRNA from Mycoplasma capricolum. Nucleic Acids Res. 9:5407-5410.

18. Karlin, S., G. M. Weinstock, and V. C. Brendel. 1995. Bacterial classifications derived from RecA protein sequence comparisons. J. Bacteriol. 177:6881-6893.

19. Macario, E. C., C. B. Dugan, and A. J. L. Macario. 1994. Identification of a grpE heat shock gene homolog in the archaeon Methanosarcina mazei. J. Mol. Biol. 240:95-101.

20. Maniloff, J., R. N. McElhaney, L. R. Finch, and J. B. Basemann (ed.). 1992. Mycoplasmas: molecular biology and pathogenesis. American Society for Microbiology, Washington, D.C.

21. Morimoto, R. I., A. Tissieres, and C. Georgopoulos (ed.). 1994. The biology of heat shock proteins and molecular chaperones. Cold Spring Harbor Laboratory Press, Cold Spring Harbor, N.Y.

22. Narberhaus, F., K. Giebeler, and H. Bahl. 1992. Molecular characterization of the dnaK gene region of Clostridium acetobutylicum, including grpE, dnaJ, and a new heat shock gene. J. Bacteriol. 174:3290-3299.

23. Oba, T., Y. Andachi, A. Muto, and S. Osawa. 1991. CGG: an unassigned or nonsense codon in Mycoplasma capricolum. Proc. Natl. Acad. Sci. USA 88:921-925.

24. Ohta, T, K. Saito, M. Kuroda, K. Honda, H. Hirata, and H. Hayashi. 1994. Molecular cloning of two new heat shock genes related to the $h s p 70$ genes in Staphylococcus aureus. J. Bacteriol. 176:4779-4783.

25. Osawa, S. A. Muto, T. Ohama, Y. Andachi, R. Tanaka, and F. Yamao. 1990. Prokaryotic genetic code. Experientia 46:1097-1106.

26. Razin, S., and E. A. Freundt. 1984. The mycoplasmas, p. 740-793. In N. R. Krieg and J. G. Holt (ed.), Bergey's manual of systematic bacteriology, vol. 1. The Williams and Wilkins Co., Baltimore, Md.

27. Razin, S. 1985. Molecular biology and genetics of mycoplasmas. Microbiol. Rev, 49:419-455.

28. Saitou, N., and M. Nei. 1987. The neighbor-joining method: a new method of reconstructing phylogenetic trees. Mol. Biol. Evol. 4:406-425.

29. Tiboni, O., P. Cammarano, and A. M. Sanangelantoni. 1993. Cloning and sequencing of the gene coding glutamine synthetase I from the archaeum 
Pyrococcus woesi: anomalous phylogenies inferred from analysis of archael and bacterial glutamine synthetase I sequences. J. Bacteriol. 175:2961-2969.

30. Weisburg, W. G., J. G. Tully, D. L. Rose, J. P. Petzel, H. Oyaizu, D. Yang, L. Mandelco, J. Sechrest, T. G. Lawrence, J. V. Etten, J. Maniloff, and C. R. Woese. 1989. A phylogenetic analysis of mycoplasmas: basis for their classification. J. Bacteriol. 171:6455 6467.
31. Wetzstein, M., U. Völker, J. Dedio, S. Löbau, U. Zuber, M. Schiesswohl, C. Herget, M. Hecker, and W. Schumann. 1992. Cloning, sequencing, and molecular analysis of the dnaK locus from Bacillus subtilis. J. Bacteriol. 174:3300-3310

32. Woese, C. R., J. Maniloff, and L. B. Zablen. 1980. Phylogenetic analysis of the mycoplasma. Proc. Natl. Acad. Sci. USA 77:494-498. 